\title{
Gestão de segurança do trabalho na indústria de papel: diagnóstico de cultura de segurança por meio de pesquisa de identificação do nível de segurança
}

Valmir Ferreira de Lima valmir_ferreiralima@hotmail.com Universidade Tecnológica Federal do Paraná (UTFPR), Ponta Grossa, Paraná, Brasil

\section{Luiz Eduardo Melo Lima} lelima@utfpr.edu.br

Universidade Tecnológica Federal do Paraná (UTFPR), Ponta Grossa, Paraná, Brasil

\begin{abstract}
RESUMO
Este trabalho apresenta informações a respeito da avaliação do desempenho da gestão de segurança praticada em uma empresa de fabricação de papel. Sendo que esta avaliação foi realizada através do diagnóstico da cultura de segurança com a aplicação da Pesquisa de Identificação do Nível de Segurança (PINS). Este estudo caracteriza-se como sendo descritivo e exploratório. Por meio de pesquisa qualitativo-quantitativa, foram realizadas análises e interpretações dos dados coletados nas avaliações da pesquisa, possibilitando o desenvolvimento das ações de conscientização dos funcionários em relação à prevenção de incidentes, atuando diretamente na disciplina do comportamento humano, guiado pelo conceito de que o trabalhador é o elemento central de todo o processo de prevenção de acidentes no trabalho. Buscou-se analisar no período de um ano a evolução da cultura de segurança, por meio das práticas realizadas na gestão de segurança. Posteriormente às análises dos dados, foi possível observar as melhorias concretas que ocorreram na cultura de segurança por meio da evolução dos estágios comportamentais e, consequentemente, uma redução do número de incidentes de trabalho, colaborando para evolução e manutenção do desempenho em segurança. Os resultados deste estudo comprovam que é de suma importância à realização de pesquisas voltadas à cultura de segurança das empresas, nos quais demonstrem a redução do número de incidentes através de ações de segurança que visam diagnosticar e proporcionar a evolução do nível de segurança dos colaboradores, considerando que estas ações são indispensáveis para promoção das condições de trabalho, nas quais previnam ou reduzam o risco de incidentes.
\end{abstract}

PALAVRAS-CHAVE: Segurança do Trabalho. Acidentes de Trabalho. Comportamento Humano. Conscientização. 


\section{INTRODUÇÃO}

A ausência de segurança nos ambientes de trabalho no Brasil, afeta negativamente a competitividade das empresas, aumentando o preço da mão de obra, refletindo no preço final dos produtos. Este fato tem causado uma preocupação para todos os segmentos produtivos.

A redução dos acidentes é um importante desafio a ser superado pelas empresas, considerando a última atualização nos dados realizada em 2013 pela Previdência Social através do seu Anuário Estatístico. Com estes dados, fica evidente que o Brasil retrocede na prevenção de acidentes, ao registrar no último ano um total de 717.911 acidentes de trabalho, que corresponde a um acréscimo de $0,55 \%$ em relação a 2012. Este cenário chama mais atenção quando relacionado ao número de mortes, sendo um total de 2.797 , que corresponde a um acréscimo de 1,05\% em relação a 2012, e o prejuízo acumula-se em toda ordem onde ocorre o incremento das despesas públicas com previdência, reabilitação profissional e saúde, reduzindo a disponibilidade de recursos para outras áreas ou levando o aumento da carga tributária sobre o contribuinte. Quando se observa o número de 1.007 acidentes com trabalhadores em 2013, atuando na fabricação de celulose, papel e produtos de papel, fica evidente a necessidade de ações (de curto, médio e longo prazo) direcionadas à gestão de segurança na prevenção de acidentes do trabalho, cujo caminho é longo, porém necessário a ser percorrido pelas empresas de base florestal (MINISTÉRIO DA PREVIDÊNCIA SOCIAL, 2015).

É importante que sejam implantadas, pelos empregadores, ações de segurança e saúde direcionadas para prevenção de acidentes decorrentes do trabalho, tanto no setor florestal quanto nos setores das indústrias de base florestal (polpa e papel, biomassa, madeira serrada, produtos florestais não madeireiros, entre outros), sempre como prioridade a manutenção da qualidade de vida de seus trabalhadores, visando garantir o aumento da produtividade, comprometimento dos trabalhadores e satisfação pessoal em trabalhar em um ambiente seguro.

Devido sua importância, as perdas relacionadas ao problema de segurança e saúde no trabalho tem se mostrado um campo de estudo viável e apresenta amplas oportunidades de pesquisa. Além disto, o estudo deste tipo de problema possui uma importante relação com as pessoas e suas atividades diárias, bem como materiais, equipamentos, meio ambiente e aspectos da produtividade da empresa (FRANZ et al., 2008, p. 139).

Portanto, é de suma importância o estudo de metodologias a serem aplicadas na gestão de segurança, no anseio de reduzir o número de incidentes na cadeia produtiva florestal, desde o viveiro, o plantio, a colheita, a logística, o pátio de madeira e a produção, até a área de expedição do produto acabado. Sendo assim, torna-se indispensável que empresas atuem diretamente com o ser humano através de capacitações, treinamentos, campanhas de conscientização, entre outros.

Segundo Leveson (2004 apud FRANZ et al., 2008, p. 140), uma gestão de segurança adequada necessita de estruturação e mecanismos que não estejam voltados para um modelo de gestão mais abrangente, ou seja, a estrutura de 
tomada de ações, os indicadores de desempenho ou as considerações sobre o posicionamento estratégico da segurança e saúde no trabalho no organograma da empresa, não são abordados conjuntamente.

Com a evolução dos níveis motivacionais dos trabalhadores, em conjunto com as frentes sindicais, a segurança do trabalho vem alcançando uma expressiva importância nas organizações, até mesmo com o reforço do atendimento aos requisitos legais. Se bem estruturadas, estas metas podem promover oportunidades dentro do planejamento estratégico, uma vez que está em evidência a avaliação da sustentabilidade da organização. Portanto, a gestão é necessária para evidenciar os critérios estratégicos das ações em segurança do trabalho.

O presente trabalho tem como principal objetivo comprovar que através do diagnóstico da cultura de segurança, com a aplicação da Pesquisa de Identificação do Nível de Segurança (PINS), é possível melhorar consideravelmente a cultura de segurança, reduzindo o número de incidentes de trabalho, colaborando para evolução e manutenção do desempenho da gestão em segurança.

\section{REVISÃO BIBLIOGRÁFICA}

Para uma melhor explanação dos conceitos relacionados com o tema tratado neste trabalho, está seção apresenta uma revisão de aspectos importantes para a segurança e saúde do trabalho: sistema de gestão em saúde e segurança do trabalho, cultura da segurança e conscientização e treinamento para alcançar os objetivos e metas.

\section{SISTEMA DE GESTÃO EM SAÚDE E SEGURANÇA DO TRABALHO}

O sistema de gestão de segurança do trabalho pode ser definido como conjunto de informações que juntas são utilizadas para constituir políticas e objetivos relacionados com a segurança no trabalho numa empresa (OHSAS, 1999, p. 9).

Conforme os conceitos apresentados por Alves e Miranda Junior (2013), os elementos disponíveis em relação à segurança e saúde do trabalho confirmam o quão é necessário que as organizações implantem um sistema de gestão aplicado à saúde e segurança do trabalho. Ainda segundo estes autores, as empresas precisam progredir com relação ao sistema de gestão de segurança, mesmo as empresas que possuem uma forte cultura de segurança e que querem atingir a excelência, mas principalmente as empresas que não possuem uma cultura forte.

Neste sentido, o planejamento, a avaliação e a gestão do sistema de segurança e saúde ocupacional da organização, servem para identificar a necessidade de habilidades especificas, bem como a necessidade de procedimentos adequados ao processo, analisar os riscos das atividades, planejar a realização das atividades e tarefas, promovendo a proteção coletiva e individual dos colaboradores (LEÃO, 2014).

Conforme descrito por Oliveira (2014), a gestão comportamental ou segurança comportamental vem sendo utilizada pela maioria das empresas, em 
especial na última década, partindo de uma série de ações destinadas à prevenção de acidentes no trabalho.

\section{CULTURA DA SEGURANÇA}

A cultura de segurança, em uma configuração simples, pode ser definida como os eventos ligados a segurança, nos quais são praticados em um determinado local, abrangendo os valores, as crenças e a maneira como ocorre esta interação com a estrutura da organização, seja por formalidade ou não (ALVES, 2014).

Para Oliveira e Milaneli (2012), uma organização demonstra que possui uma cultura de prevenção de acidentes quando adota ações que vão além do comprimento de requisitos legais, ou seja, com ações que priorizam o trabalhador.

Ainda neste sentido, fazem parte da cultura da organização, a forma como são tomadas as decisões, o modo de liderança e a definição de valores, bem como a prática por parte de seus integrantes, sendo este um conjunto de ações preventivas nas quais influenciam sobre a maneira em que as pessoas aprendem o sentido de seguro ao expor-se ao risco durante sua atividade. Deste modo, o comportamento do indivíduo é influenciado pelo contexto (BLEY, 2011).

A gestão de segurança atua nos valores compartilhados e nas crenças do conjunto de pessoas de uma organização que interagem com a sua estrutura, bem como com os sistemas de controle que possuem para produzir modelos comportamentais escritos ou não relacionados à saúde e segurança (ALVES e MIRANDA JUNIOR, 2013).

Partindo deste princípio, torna-se evidente que a adoção de qualquer padrão de gestão deve-se levar em consideração a questão cultural da organização, evitando que o padrão almejado se torne apenas um conjunto de ações de segurança que não possui correlação nenhuma com a realidade vivenciada na empresa (MORAES JUNIOR, 2003).

\section{DIAGNÓSTICO DA CULTURA DE SEGURANÇA}

Conforme descrito por Cardella (1999), o diagnóstico de segurança é o fruto do estudo de quem tem por anseio reconhecer o estado de segurança da organização. $O$ estado de segurança estudado é confrontado com um padrão e o desvio serve de insumo para elaboração das ações preventivas. Aspectos comportamentais de uma organização podem ser mapeados, classificados e denominados como estágios de segurança, sendo esta fase essencial para o processo da gestão de cultura em segurança, possibilitando a estratégia de ações de prevenção.

Para Alves e Miranda Junior (2013), pesquisas de clima e cultura de segurança devem ser feitas buscando identificar o progresso cultural que ocorre na empresa, podendo ser realizadas por meio de técnicas qualitativas, de grupos específicos ou de forma quantitativa por meio de questionários. Além disto, conforme descrito por estes autores, o momento complexo no qual está inserida 
as organizações modernas demanda que se opere uma gestão que possibilite a análise de informações complexas e numerosas para a tomada de decisões.

Segundo Oliveira (2014), é possível perceber a evolução da segurança e da saúde ocupacional no Brasil nas últimas décadas pela análise de inúmeras variáveis, das quais se destacam os estágios descritos pela curva de Bradley, conforme apresentado na Figura 1. Sendo estes estágios denominados por reativo, dependente, independente e interdependente, sendo os dois últimos os estágios mais evoluídos. Ainda segundo o trabalho deste autor, a evolução dos estágios reativo e dependente para os estágios mais evoluídos, independente e interdependente, foi considerada admissível em muitas organizações. Essa evolução possibilitou a organização alcançar uma redução dos índices de acidentes no trabalho de forma sistemática, bem como entender e tratar o tema sobre outro ponto de vista.

Figura 1 - Classes comportamentais ou estágios da curva de Badley que podem ser identificados a partir da aplicação da PINS
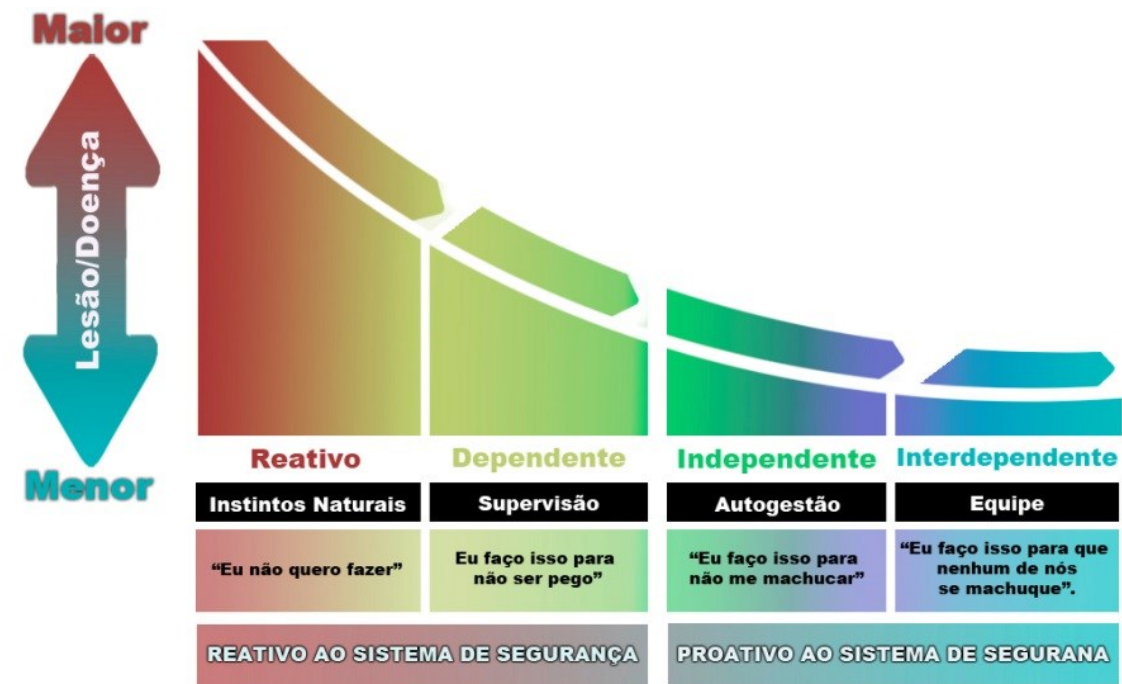

Fonte: Adaptado de DuPont (2015)

Em uma cultura de segurança madura, a segurança é um conceito aplicável que é realmente sustentável, com taxas de lesões praticamente nulas. As pessoas sabem que podem agir conforme necessário para trabalhar com segurança, apoiando e desafiando umas às outras. As decisões são tomadas no nível apropriado e as pessoas vivem de acordo com estas decisões. A organização como um todo obtém benefícios comerciais significativos que se refletem em mais qualidade, maior produtividade e lucros mais elevados (DUPONT, 2015).

Segundo Pinto (2015), a busca pelo compromisso com o acidente zero, não pode ser tratado apenas como um objetivo estatístico, mas como uma questão mútua de valorização do trabalhador e da evolução dos estágios de segurança, possibilitando a redução do número de acidentes no trabalho, através do qual a utilização da curva de Bradley (conforme apresentado na Figura 1) possibilita a verificação. 
Por definição da OHSAS (1999, p. 10), as pessoas devem possuir competência para exercer suas atividades nas quais impactam na saúde e segurança ocupacional. Esta aptidão deverá ser determinada com parâmetros voltados para a educação, treinamento e experiência. Conforme Tavares (2008), todo empregado deverá passar por treinamentos onde seja possível a compreensão de que a segurança é também uma responsabilidade particular.

Para Araújo (2010), o treinamento é o conjunto de metodologias nas quais produzem ou se aprimoram as noções para o trabalho. Adicionalmente, de acordo com Bley (2011, p. 11):

Capacitar e desenvolver pessoas para que se tornem competentes em pensar, sentir e agir cuidando de si mesmas, dos outros e deixando-se cuidar pelos outros parecem ser o grande objetivo da segurança baseada no comportamento humano. Para que isso se torne realidade em nosso país, onde o cenário de doenças e acidentes de trabalho está longe do que se considerado aceitável (se é que isso existe), é preciso que governantes e dirigentes de organizações revejam suas políticas de gestão de pessoas, especialmente no que diz respeito às estratégias de treinamento $\mathrm{e}$ desenvolvimento (BLEY, 2011 p. 11).

Ainda de acordo com Bley (2011), se a prevenção de acidentes do trabalho pode ser considerada um método comportamental, instruir-se conceber a possibilidade de incidirem comportamentos expressivos para a segurança dos trabalhadores. Deste modo, é proeminente que seja analisado a importância da metodologia de ensino e aprendizado com foco na prevenção de acidentes do trabalho. As melhores tecnologias de trabalho são ineficazes nas mãos de trabalhadores desmotivados ou sem a capacitação adequada para executar suas atividades.

Com base na abordagem de Oliveira e Milaneli (2012), os programas de prevenção de acidentes baseados no comportamento, deverão preferencialmente ser conduzidos por profissionais especializados e com um foco predominante em psicologia do trabalho.

As técnicas empregadas no processo de evolução são psicodinâmicas, nas quais ocorre a participação e interação permitindo melhor detenção dos conteúdos. Levando em consideração que o ser humano retém conhecimento por repetição e constância, podem ser realizadas dinâmicas, exercícios e situações nas quais o trabalhador possa vivenciar conhecimentos e evidenciar que existe direcionamento ao comportamento seguro. A mudança de paradigmas pode ser tratada como um campo ainda não explorado, onde ocorre o ingresso de pessoas em uma empresa nas quais precisam passar pelo processo de treinamento, porém, também necessitam ser educadas (ALVES e MIRANDA JUNIOR, 2013). 


\section{METODOLOGIA}

A metodologia adotada para a realização do presente estudo está dividida em quatro partes, a saber: classificação da pesquisa, sistema de gestão em saúde e segurança do trabalho, coleta de dados e tratamento e análise de dados.

\section{CLASSIFICAÇÃO DA PESQUISA}

O desenvolvimento deste trabalho foi realizado por meio das aplicações de ações projetadas e praticadas na empresa objeto de estudo. A empresa está localizada no município de Arapoti, estado do Paraná, a qual abrange uma área total de $794.500 \mathrm{~m}^{2}$, sendo $68.990,07 \mathrm{~m}^{2}$ de área construída, tendo como ramo de atividade a fabricação de papel.

Foi estudada a gestão de segurança no trabalho com base na conscientização, direcionada aos colaboradores das diversas áreas da fábrica, tendo como finalidade verificar o desempenho da gestão de segurança e evidenciar o fortalecimento da cultura de segurança.

Segundo Gil (2002, p. 41), a pesquisa descritiva e exploratória busca expor as particularidades de uma equipe, fato ou até mesmo a afirmativa de semelhanças, conforme o estilo de técnicas associadas para coleta de informações. Estas pesquisas têm como objetivo proporcionar maior familiaridade com o problema, com vistas a torná-lo mais explícito ou a constituir hipóteses. Pode-se dizer que estas pesquisas têm como objetivo principal o aprimoramento de ideias ou a descoberta de intuições. Seu planejamento é, portanto, bastante flexível, de modo que possibilite a consideração dos mais variados aspectos relativos ao fato estudado.

A abordagem para a análise realizada neste trabalho é também classificada como quantitativa, pois explana os dados em formatos de números. Os dados interpretados por meio de julgamento das respostas alcançadas como qualitativa porque se estuda um fato característico com dedicação, sendo com aspecto indutivo, tendo como ambiente adequado de coleta de dados (SILVA e MENEZES, 2001). A pesquisa utilizou dados reais quantitativos, conforme apresentado na Tabela 1, porém recaindo sobre a questão qualitativa devido à pesquisa de identificação do nível segurança comportamental dos colaboradores.

Tabela 1 - Funções estudadas por gerência na empresa objeto de estudo

\begin{tabular}{cc}
\hline Gerência & Funções estudadas \\
\hline Supply chain & 14 \\
Papel e acabamento & 25 \\
Fibras e utilidades & 11 \\
Engenharia e manutenção & 25 \\
Tecnologia da informação / controladoria & 12 \\
Qualidade e meio ambiente & 6 \\
Recursos humanos & 8 \\
Diretoria industrial & 2 \\
\hline
\end{tabular}

Fonte: Pesquisa de campo (2013). 
Para a realização deste estudo elaborou-se um questionário, o qual foi utilizado para a pesquisa, denominada de Pesquisa de Identificação do Nível de Segurança (PINS), que por sua vez está associada ao enfoque comportamental. De acordo com Gil (2002, p. 129):

Construir um questionário consiste basicamente em traduzir os objetivos da pesquisa em questões especificas. As respostas a essas questões é que irão proporcionar os dados requeridos para testar as hipóteses ou esclarecer o problema da pesquisa. As questões constituem, pois, o elemento fundamental do questionário (GIL, 2002, p. 129).

Sendo assim, o questionário aplicado pela empresa abordou questões direcionadas ao comportamento humano em relação ao sistema de segurança estabelecido pela empresa, buscando identificar as classes comportamentais (estágios) as quais ocorrem à reatividade, dependência, independência e a interdependência em relação ao sistema de segurança.

\section{COLETA DE DADOS}

Tendo como metodologia a realização de entrevistas com a liderança de cada área, sempre com a presença de um responsável pela área de recursos humanos ou departamento de saúde e segurança do trabalho, o psicólogo contratado efetuou o diagnóstico por meio de formulário a fim de coletar o máximo de informações necessárias para então definir as classes comportamentais (estágios) existente na empresa.

A aplicação de questionário da Pesquisa de Identificação do Nível de Segurança (PINS) foi realizada pelas lideranças em duas etapas, onde se avaliou $100 \%$ dos colaboradores, de acordo com o fluxograma apresentado na Figura 2. A primeira etapa da pesquisa ocorreu nos meses de janeiro e fevereiro de 2014 (com 314 funcionários entrevistados), onde foram realizadas as entrevistas individuais com todos os gestores, com o intuito de se avaliar o estado inicial da gestão em saúde e segurança do trabalho. A segunda etapa, ou seja, o monitoramento da evolução da gestão em saúde e segurança do trabalho (com 327 funcionários entrevistados) foi realizado em outubro do mesmo ano, repetindo-se o processo de entrevistas individuais com gestores buscando analisar a evolução que ocorreu dentro das classes comportamentais (estágios). 
Figura 2 - Fluxograma de aplicação da PINS para coleta de dados

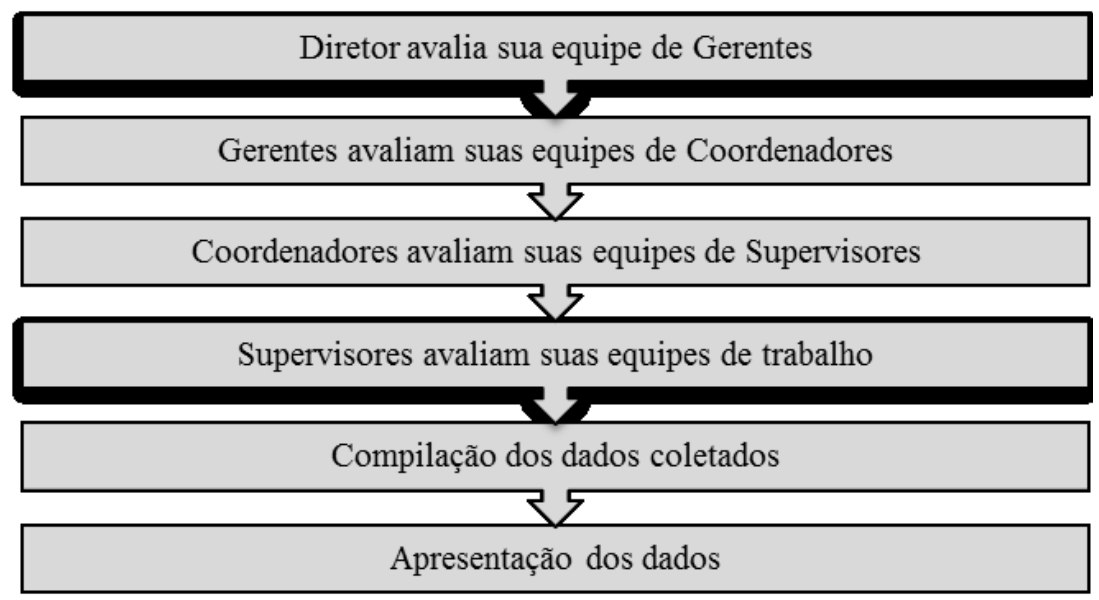

Fonte: Autoria própria (2017)

\section{TRATAMENTO E ANÁLISE DE DADOS}

Através da pesquisa aplicada na empresa, foi possível diagnosticar as classes comportamentais (estágios) nas quais se encontravam os funcionários, possibilitando definir as ações a serem desenvolvidas pela empresa, com o objetivo de atingir o nível de segurança necessário para prática diária da segurança como valor da empresa. A meta foi evidenciar a evolução dos comportamentos para as classes independente e interdependente, desenvolvendo o estado de consciência individual e coletiva do comportamento seguro, ou seja, o desenvolvimento do estado de alerta, elemento este importante para a redução do número de acidentes. Após a identificação dos trabalhadores interdependentes, estes foram indicados como membros de uma equipe de Sentinelas da Vida, que atuam nas ações de prevenção.

\section{RESULTADOS E DISCUSSÕES}

Nesta seção são apresentados os resultados da Pesquisa de Identificação do Nível de Segurança (PINS), realizada em duas etapas (PINS-1 e PINS-2), bem como a análise e discussão destes resultados. Também são apresentadas as ações realizadas pelo sistema de gestão em saúde e segurança do trabalho, após o 10 diagnóstico, bem como a análise e apresentação da redução dos números de incidentes por meio das ações realizadas pelo sistema de gestão em saúde e segurança do trabalho, após a avaliação do 2 o diagnóstico em relação ao 1 으 diagnóstico.

\section{ANÁLISE DOS RESULTADOS DA PINS-1 E AÇÕES REALIZADAS APÓS O 1ำ DIAGNÓSTICO}

A partir dos resultados apresentados no Gráfico 1, é possível analisar e interpretar os dados coletados nas avaliações da PINS-1, relacionado ao 1 o diagnóstico da cultura de segurança, realizada em janeiro e fevereiro de 2014, 
com a finalidade de avaliar o estado inicial do sistema de gestão em saúde e segurança do trabalho na empresa.

Gráfico 1 - Resultados da 1ạ Pesquisa de Identificação do Nível de Segurança (PINS-1)

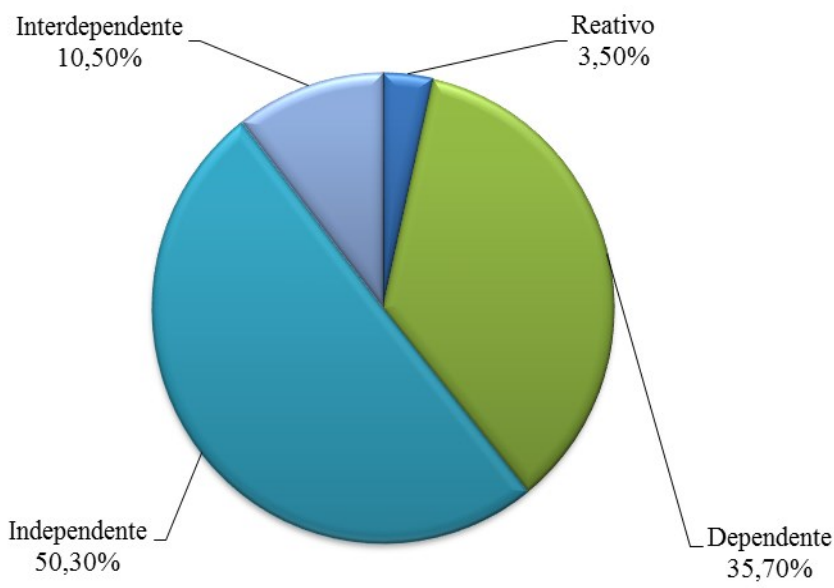

Fonte: Autoria própria (2017).

Após análise e interpretação dos dados coletados no período da 1a pesquisa (PINS-1) é possível observar que em relação aos 314 funcionários avaliados têmse os seguintes tipos de comportamento (estágios):

a) $3,50 \%$ dos colaboradores praticam a segurança por que alguém determina, pois, a segurança não é vista como algo importante;

b) $35,70 \%$ dos colaboradores praticam a segurança para não ser pego, necessitando de supervisão para que a prevenção ocorra;

c) $50,30 \%$ dos colaboradores praticam a segurança para não se machucar, possuindo autogestão e aceitação em seguir as regras de segurança;

d) $10,50 \%$ dos colaboradores praticam a segurança para que ninguém da equipe ou da empresa se machuque, possuindo dedicação e compromisso para com as normas de segurança.

\section{Ações Realizadas Pelo Sistema de Gestão em Saúde e Segurança do Trabalho}

As ações realizadas pelo sistema de gestão em saúde e segurança do trabalho após o 10 diagnóstico foram acompanhadas por um psicólogo contratado, juntamente com a área de recursos humanos e o departamento de saúde e segurança do trabalho da empresa. Estas ações são descritas a seguir:

a) Desenvolvimento das 8 Regras Essenciais de Segurança - Por meio de análise de legislações aplicáveis, as regras foram elaboradas juntamente com os funcionários, buscando seu entendimento e aplicabilidade em todos os níveis hierárquicos da empresa, servindo como alicerce para a cultura de segurança como valor na empresa;

b) Regras Específicas de Segurança - Para orientar e fortalecer a cultura de segurança dos funcionários envolvidos nas atividades de segurança, por meio da prevenção e de acordo com os perigos e riscos específicos de cada setor, as Regras Específicas de Segurança foram desenvolvidas pelos trabalhadores; 
c) Treinamentos e Palestras - Foram realizados diversos treinamentos e palestras com foco na mudança de comportamento, os quais contribuíram no processo de desenvolvimento da cultura de segurança nos funcionários;

d) Sentinelas da Vida - Após a Pesquisa de Identificação do Nível de Segurança (PINS), foram identificados os funcionários que pertenciam ao estágio de interdependência de cada área, desenvolvendo-se a equipe de Sentinelas da Vida, cujas auxiliam nas ações de segurança. A fim de evidenciar o trabalho realizado pelas Sentinelas da Vida foram desenvolvidas camisetas padronizadas para equipe;

e) SIPAT - Atuou-se nas ações preventivas de segurança para a redução de incidentes, realizando campanhas de conscientização junto aos funcionários por meio da SIPAT, com o envolvimento das famílias destes funcionários. A SIPAT foi idealizada e organizada com o objetivo de sensibilizar e aproximar o funcionário e sua família para com as metas de segurança da empresa;

f) Observação de Comportamento em Segurança - O processo de segurança baseado em observação de comportamento em segurança, que visa obter um local de trabalho saudável e seguro, tem como princípio que se o comportamento é "observável", ele também pode ser "gerenciado" e conduzido para uma direção mais segura;

g) Percepção dos Riscos - Foram desenvolvidas atividades visando melhorar a percepção dos riscos, nas quais era simulada a realização de tarefas que apresentavam situações de diversos tipos de riscos no seu desenvolvimento, sendo necessária por parte dos funcionários à identificação e descrição por meio de formulário a maneira segura;

h) Desenvolvimento de Marketing/Comunicação - Para Tavares (2008), o emprego de cartazes, apresentações, periódicos de segurança, filmes, sugestões, revistas especializadas, etc., auxiliam na conscientização do trabalhador em relação à segurança do trabalho. Neste sentido, foram desenvolvidos materiais para orientação dos funcionários com base na proposta do fortalecimento da cultura de segurança tais como banners, faixas, adesivos, bonecos e uma cartilha com as 8 Regras Essenciais de Segurança.

\section{ANÁLISE DOS RESULTADOS DA PINS-2 E EVOLUÇÃO DO NÍVEL DE GESTÃO DE SEGURANÇA}

Os resultados expostos no Gráfico 2 possibilitam a análise dos dados coletados nas avaliações da PINS-2, relacionado ao 2임 diagnóstico da cultura de segurança, realizada em outubro de 2014, com o objetivo de verificar a evolução do nível de gestão de segurança na empresa. 
Gráfico 2 - Resultados da 2a Pesquisa de Identificação do Nível de Segurança (PINS-2)

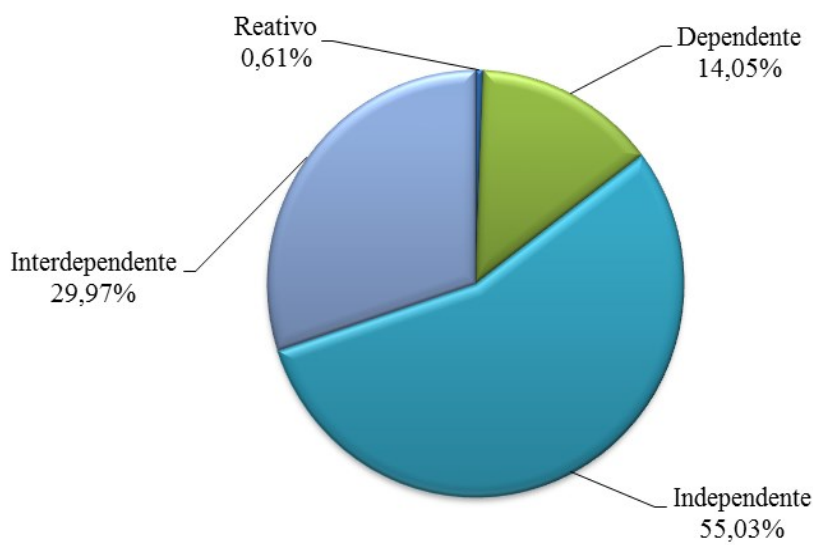

Fonte: Autoria própria (2017).

A partir da análise dos dados coletados no período da 2a pesquisa (PINS-2) é possível identificar que ocorreu uma evolução em relação à redução dos valores percentuais dos colaboradores classificados nos comportamentos reativos e dependentes, relacionada às ações realizadas pelo sistema de gestão em saúde e segurança no trabalho. De forma geral, foi possível identificar melhorias expressivas, concretas e mensuráveis em relação à cultura de segurança na organização no período analisado. Para demonstrar esta evolução, é possível apresentar, por meio da análise e da interpretação dos dados, a evolução dos estágios comportamentais no 2 으 diagnóstico (PINS-2) em relação ao 1 o diagnóstico (PINS-1), conforme observado no Gráfico 3.

Gráfico 3 - Comparativo da evolução dos estágios comportamentais na cultura de segurança da empresa

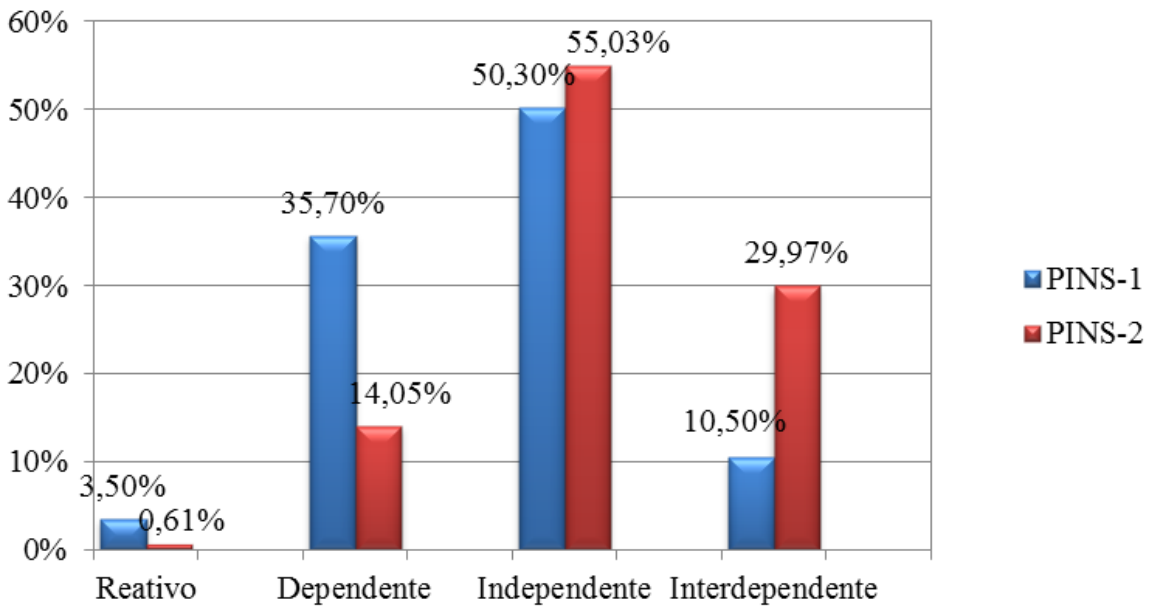

Fonte: Autoria própria (2017).

A partir dos resultados apresentados no Gráfico 3, é possível observar que em relação aos 327 funcionários avaliados têm-se as seguintes alterações nos tipos de comportamento (estágios): 
a) Redução de $3,50 \%$ para $0,61 \%$ (equivalente a uma redução de $2,80 \%$ do valor percentual) dos colaboradores que praticavam a segurança por que alguém determina, pois, a segurança não é vista como algo importante;

b) Redução de $35,70 \%$ para $14,05 \%$ (equivalente a uma redução de $21,65 \%$ do valor percentual) dos colaboradores que praticavam a segurança para não ser pego, necessitando de supervisão para que a prevenção ocorra;

c) Aumento de $50,30 \%$ para $55,03 \%$ (equivalente a um aumento de $5,06 \%$ do valor percentual) dos colaboradores que praticavam a segurança para não se machucar, possuindo autogestão e aceitação em seguir as regras de segurança;

d) Aumento de $10,50 \%$ para $29,97 \%$ (equivalente a um aumento de $19,40 \%$ do valor percentual) dos colaboradores que praticavam a segurança para que ninguém da equipe ou da empresa se machuque, possuindo dedicação e compromisso para com as normas de segurança.

\section{Análise da Redução dos Números de Incidentes por Meio das Ações Realizadas da Gestão de Segurança}

A evolução dos estágios reativo e dependente para os estágios mais evoluídos, independente e interdependente, foi visível dentro do processo de gestão de segurança do trabalho adotado pela empresa. Essa evolução, entre outros benefícios, resultou numa redução sistemática dos índices de incidentes no trabalho por gerência, entre os anos de 2013 e 2014, conforme resultados apresentados no Gráfico 4.

Gráfico 4 - Comparativo da redução dos incidentes por gerência na empresa

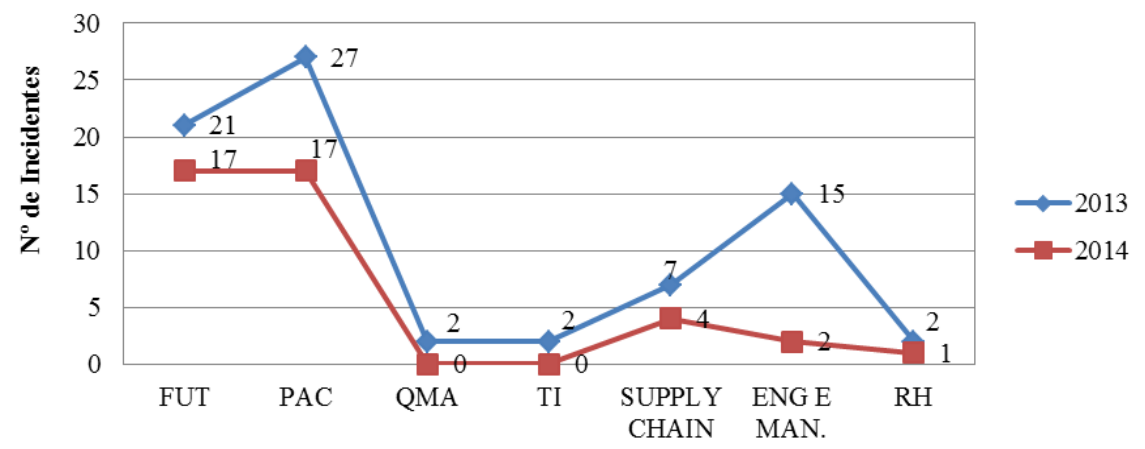

Fonte: Autoria própria (2017).

É possível observar a partir do Gráfico 4, que a área de Fibras e Utilidades (FUT) reduziu um total de 4 ocorrências, que corresponde a uma redução de 19,0\% das ocorrências. O mesmo foi possível identificar na área de Engenharia e Manutenção (ENG E MAN.) que apresentou a redução de 13 ocorrências, que corresponde a uma redução de $86,7 \%$ das ocorrências. Outra área que apresentou uma significativa redução foi área de Papel e Acabamento (PAC) com a redução de 10 ocorrências, que corresponde a uma redução de $37,0 \%$ das ocorrências. A área de Supply Chain consolidou uma redução total de 3 ocorrências, que corresponde a uma redução de $42,9 \%$ das ocorrências. Na área de Recursos Humanos (RH) foi possível identificar a redução total de 1 ocorrência, que corresponde a uma redução de 50\% das ocorrências. Nas áreas 
de Qualidade e Meio Ambiente (QMA) e Tecnologia da Informação (TI), foi possível identificar a redução total de 2 ocorrências para cada gerência, que corresponde à redução de $100 \%$ das ocorrências.

Os números apresentados nesta análise demonstram que está ocorrendo um processo de evolução no sentido de se buscar o comprometimento de todos os envolvidos em termos de valores, atitudes e comportamentos direcionados a redução do número de incidentes, através do aumento do desempenho em segurança, por meio da postura baseada na prevenção adotada pelos trabalhadores, como consequência das ações aplicadas no sistema de gestão em saúde e segurança do trabalho na empresa objeto de estudo.

\section{CONCLUSÕES E RECOMENDAÇÕES}

A partir dos dados avaliados neste trabalho, foram observadas melhorias concretas na cultura de segurança e, consequentemente, uma redução significativa no número de incidentes de trabalho no período avaliado na empresa de fabricação de papel. Tal evolução dos estágios comportamentais, só foi possível ser verificada em função do diagnóstico do nível de segurança realizado por meio da Pesquisa de Identificação do Nível de Segurança (PINS), que possibilitou a identificação dos pontos críticos a serem melhorados e a partir disso foram desenvolvidas as ações necessárias para aumentar o desempenho de segurança do trabalho na empresa.

Conforme observado neste trabalho, identificar a cultura de segurança, bem como os estágios comportamentais, garante que os investimentos aplicados na área de segurança sejam direcionados de acordo com as necessidades especificas de cada empresa, resultando numa maior eficiência da aplicação destes investimentos e a obtenção dos resultados esperados.

Deste modo, a aplicação do diagnóstico da cultura de segurança por meio da avaliação das classes ou estágios comportamentais, utilizando a Pesquisa de Identificação do Nível de Segurança (PINS) como ferramenta, é uma metodologia recomendada na gestão de segurança das empresas, independente do ramo de atividade, podendo colaborar para evolução e manutenção do desempenho em segurança a partir da execução de ações planejadas. 


\title{
Work safety management in the paper industry: safety culture diagnostics through identification survey security level
}

\author{
ABSTRACT
}

This paper presents information about the performance evaluation of the safety management practiced in a paper manufacturing company. This evaluation was performed through the safety culture diagnosis with the application of the Security Level Identification Survey. This study is characterized as being descriptive and exploratory. Through qualitative/quantitative research, were carried out analysis and interpretation of data collected in the survey ratings, allowing the development of the employee awareness campaigns regarding incident prevention, acting directly in the course of human behavior, guided by the concept that the worker is the central element of the process of accidents prevention at work. It was sought to examine the one-year period the evolution of safety culture through the practices carried out in safety management. After the data analysis, it was possible to observe the concrete improvements that have occurred in the safety culture through the development of behavioral stages and consequently reducing the number of work incidents, contributing to development and maintenance of safety performance. The results of this study prove that is extremely important to conduct research focused on the safety culture of the companies in which to demonstrate a reduction in incident number through security actions aimed at diagnosing and provide the evolution of employee security level considering that these actions are essential to promote working conditions, in which prevent or reduce the risk of incidents.

KEYWORDS: Workplace Safety. Safety Culture. Human Behavior. Awareness. 


\section{REFERÊNCIAS}

ALVES, J. L. L. Comportamento de risco. Revista Proteção, n. 1, 2014. Disponível em: <http://www.protecao.com.br/edicoes/1/2014/AAjj>. Acesso em: 11 abr. 2015.

ALVES, J. L. L.; MIRANDA JUNIOR, L. C. Mudança cultural orientada por comportamento: elementos para uma cultura de saúde, segurança, confiabilidade e produtividade, atuando com as pessoas. $1^{\text {a }}$ ed. Rio de Janeiro: Qualitymark, 2013.240 p.

ARAÚJO, W. T. Manual de segurança do trabalho. São Paulo: DCL - Difusão Cultural do Livro, 2010. 453 p.

BLEY, J. Comportamento seguro: a psicologia da segurança no trabalho e a educação para a prevenção de doenças e acidentes. Versão e-book, 2011. Disponível em: <http://temseguranca.com/livro-gratuito-sobre-comportamentoseguro/>. Acesso em: 10 abr. 2015.

CARDELLA, B. Segurança no trabalho e prevenção de acidentes: uma abordagem holística: segurança integrada à missão organizacional com produtividade, qualidade, preservação ambiental e desenvolvimento de pessoas. São Paulo: Atlas, 1999. 254 p.

DUPONT. A curva de Bradley da DuPont. Disponível em: $<$ http://www.dupont.com.br/produtos-e-servicos/consulting-services-processtechnologies/segurancadotrabalho/usos-e-aplicacoes/bradley-curva.html>. Acesso em: 1 ago. 2015.

FRANZ, L. A. S.; AMARAL, F. G.; MARTINS, P. M. F. Modelos de gestão da segurança e saúde no trabalho: uma revisão sobre as práticas existentes e suas características. Revista Gestão Industrial, v. 4, n. 4, p. 138-154, 2008.

GIL, A. C. Como elaborar projetos de pesquisa. 4a ed. São Paulo: Atlas, 2002. 175 p.

LEÃO, D. Heinrich e Bird: prevenção e controle de perdas. Escola da prevenção. 2014. Disponível em: <http://escoladaprevencao.com/colunistas/heinrich-e-birdprevencao-e-controle-de-perdas.html>. Acesso em: 10 abr. 2015.

LEVESON, N. A new accident model for engineering safer systems. Safety Science, v. 42 , n. 4 , p. 237-270, 2004. 
MINISTÉRIO DA PREVIDÊNCIA SOCIAL. Anuário estatístico de acidentes do trabalho 2013. 2015. Disponível em: <http://www.previdencia.gov.br/aeat2013/>. Acesso em: 1 jun. 2015.

MORAES JUNIOR, C. P. Sistema de gestão - assunto da moda. Artigo do site de segurança no trabalho "AreaSeg.com" de Carlos Alberto Marangon. Disponível em: <http://www.areaseg.com/artigos/cosmo9.zip>. Acesso em: 25 abr. 2015.

OLIVEIRA, C. A. D.; MILANELI, E. Manual prático de saúde e segurança do trabalho. 2a ed. São Caetano do Sul: Yendis, 2012. 464 p.

OLIVEIRA, J. C. Evolução e maturidade em segurança e saúde no trabalho: a dificultosa transição dos estágios básicos de gestão de SST (primeiro e segundo) para os estágios mais elevados (terceiro e quarto). 2014. Disponível em: <https://eustaquiodiniz.files.wordpress.com/2014/02/evoluc3a7c3a3o-ematuridade-em-sst-fevereiro-2014.pdf >. Acesso em: 1 jun. 2015.

OHSAS. 18001: Sistemas de gestão de segurança e saúde ocupacional especificação. Reino Unido: British Standards Institution, 1999, 30 p.

PINTO, C. Prevenção e segurança. 2015. Disponível em: <http://carinapinto1111750.wixsite.com/pesee/singlepost/2015/05/20/Preven\%C3\%A7\%C3\%A3o-e-Seguran\%C3\%A7a>. Acesso em: 10 ago. 2015.

SILVA, E. L.; MENEZES, E. M. Metodologia da pesquisa e elaboração de dissertação. 3ạ ed. Florianópolis: Laboratório de Ensino a Distância da UFSC, 2001.

TAVARES, J. C. Noções de prevenção e controle de perdas em segurança do trabalho. 6a ed. São Paulo: Senac, 2008. 143 p. 
Recebido: 05 maio. 2017

Aprovado: $18 \mathrm{dez} .2017$

DOI: 10.3895/gi.v13n4.5877

Como citar:

LIMA, V. F.; LIMA, L. E. M. Gestão de segurança do trabalho na indústria de papel: diagnóstico de cultura de segurança por meio de pesquisa de identificação do nível de segurança. R. Gest. Industr., Ponta Grossa, v.

13, n. 4, p. 205-222, out./dez. 2017. Disponível em: <https://periodicos.utfpr.edu.br/rgi>. Acesso em: XXX

Correspondência:

Valmir Ferreira de Lima

Rua Felicindo Barrios Trigo, 243, Jardim Ceres, Arapoti, Paraná, Brasil.

Direito autoral: Este artigo está licenciado sob os termos da Licença Creative Commons-Atribuição 4.0

Internacional.

cC) (i) 